\title{
Single-Trace Side-Channel Attacks on Scalar Multiplications with Precomputations
}

\author{
Kimmo Järvinen ${ }^{1}$ and Josep Balasch ${ }^{2}$ \\ 1 Aalto University, Department of Computer Science \\ kimmo.jarvinen@aalto.fi \\ 2 KU Leuven ESAT/COSIC and iMinds \\ josep.balasch@esat.kuleuven. be
}

\begin{abstract}
Single-trace side-channel attacks are a serious threat to elliptic curve cryptography in practice because they can break also cryptosystems where scalars are nonces (e.g., ECDSA). Previously it was believed that single-trace attacks can be avoided by using scalar multiplication algorithms with regular patterns of operations but recently we have learned that they can be broken with correlation tests to decide whether different operations share common operands. In this work, we extend these attacks to scalar multiplication algorithms with precomputations. We show that many algorithms are vulnerable to our attack which correlates measurements with precomputed values. We also show that successful attacks are possible even without knowledge of precomputed values by using clustering instead of correlations. We provide extensive evidence for the feasibility of the attacks with simulations and experiments with an 8-bit AVR. Finally, we discuss the effectiveness of certain countermeasures against our attacks.
\end{abstract}

\section{Introduction}

Elliptic curve cryptography (ECC) $[36,39]$ has gained huge popularity in secure embedded systems because it offers high cryptographic security with short key sizes and relatively low computational requirements. The most important operation in ECC is the scalar multiplication $Q=k P$ where $Q$ and $P$ are points on an elliptic curve and $k$ is an integer. The scalar $k$ is typically secret and must remain unknown to attackers in order to maintain the security.

Side-channel attacks on ECC have gained significant amounts of research interest $[17,18]$. We focus on passive attacks where an attacker only observes a cryptographic device under its normal operation. They can be categorized into two classes: single-trace attacks, which use measurements from only one scalar multiplication, and multi-trace attacks, which utilize statistical methods onto multiple scalar multiplications sharing the same scalar. Single-trace attacks are much more serious threats because in most ECC protocols (e.g., ECDSA [44]) scalars are nonces, i.e., short-term secrets that change at each protocol execution. This prevents the attacker from obtaining multiple traces with the same scalar and nullifies multi-trace attacks. However, the attacker may still often launch a 
repeated single-trace attack where she can acquire multiple traces with different scalars. We focus on (repeated) single-trace attacks in this paper.

The simplest algorithm for computing scalar multiplications is the doubleand-add, where a point doubling $(2 Q)$ is computed for every bit of $k$ but a point addition $(Q+P)$ only when a bit is one. The double-and-add succumbs even to simple power analysis (SPA) where measurement traces are observed visually. It is relatively easy to protect against these attacks by adopting the so-called atomicity principle. An atomic implementation has a regular pattern of operations which does not depend on $k$. This can be achieved, e.g., by using dummy operations (e.g., double-and-add-always [13]), a regular scalar multiplication algorithm (e.g., Montgomery's ladder [35, 41]), balancing point addition and doubling formulea (e.g., $[9,25])$, or using a unified point addition and doubling formula (e.g., [7]). Prior to recent advanced single-trace attacks, these principles were considered sufficient to protect against single-trace attacks.

The history of advanced single-trace attacks began in 2001 with Walter's Big Mac attack [52] on exponentiations, where the attacker targets partial multiplications computed in each long integer multiplication of an exponentiation. In 2010, Clavier et al. [11] introduced a single-trace attack on a larger class of exponentiation algorithms called horizontal correlation analysis by utilizing ideas from the Big Mac attack together with correlation power analysis. Correlation power analysis was originally introduced against secret-key cryptography by Brier et al. [8] in 2004 and it was already earlier applied in multi-trace attacks on public-key algorithms by Amiel et al. [1] in 2007. In 2013, Bauer et al. [4] (see [5] for an extended version) combined the ideas of [11] with Moradi et al.'s collision correlation analysis [42] and obtained a very powerful single-trace attack which thwarts many of the state-of-the-art countermeasures. Recently, further works have built on the ideas of improving Big Mac [15] and collision correlations [29]. Another trend of attacks uses clustering algorithms to launch a single-trace attack on ECC. A clustering attack was shown to break an ECC hardware implementation without any profiling or leakage models by Heyszl et al. [31]; Sprecht et al. [49] later improved this attack. In addition to these, also local electromagnetic measurements have been shown to offer means to launch successful single-trace attacks, e.g., by Heyszl et al. [32].

Precomputations based on $P$ are commonly used to speed up scalar multiplications, but they play a role also in side-channel security. Even if SPA can distinguish point additions from point doublings, it is incapable of distinguishing operands used in point additions. For this reason, the double-and-add algorithm with, e.g., the width- $w$ non-adjacent form ( $w$-NAF), where digits are from $\left\{0, \pm 1, \pm 3, \ldots, \pm\left(2^{w-1}-1\right)\right\}$ (see, e.g., [28]), offers better protection against sidechannel attacks than a binary expansion, where digits are from $\{0,1\}$. Nevertheless, direct use of, e.g., $w$-NAF still leaks a lot of information about the scalar and cannot be considered side-channel secure. Fully regular patterns of operations can be achieved with atomic scalar multiplication algorithms with precomputations which typically combine side-channel security with efficiency (see, e.g., [19, $30,45,46])$. Such algorithms have been recently used for side-channel protected 
lightweight hardware implementations, e.g., in $[47,48]$ as well as fast software, e.g., in [14]. In the light of new advanced single-trace attacks, there have been doubts about the security offered by these algorithms (see, e.g., [3, 31, 47]).

We show that these doubts are well grounded, at least for processors with small word sizes. Hence, our paper has significance, in particular, for lightweight implementations of ECC. Such implementations typically implement ECC on 8bit or 16-bit processors (see, e.g., [10,27,37,38,43,50]) or utilize datapaths with small multipliers (e.g., 16-bit multipliers used in [47,48]). The contributions of this paper can be summarized as follows:

- We extend existing correlation-based single-trace attacks (in particular, [4, 5, $15,29,52])$ to scalar multiplications with precomputations. While this type of correlation attacks have been conjectured to form a serious threat to scalar multiplication algorithms with precomputations already before, we are not aware of any works that would have studied this in depth before this paper.

- We show that a clustering-based attack allows the attacker to successfully attack these algorithms even without relying on a specific leakage model and with unknown precomputed values.

- We provide comprehensive evidence about the practicability of these attacks through simulations and experiments with an 8-bit AVR (ATMega163).

- We present a summary of countermeasures.

The paper is organized as follows: Sect. 2 discusses scalar multiplication algorithms that we target with our attacks. Sect. 3 describes the attack and its two variants. Sect. 4 presents simulation results and Sect. 5 presents experiments with the 8-bit AVR. Sect. 6 discusses countermeasures against the attacks. Finally, we draw conclusions and discuss topics for future research in Sect. 7.

\section{Preliminaries}

We assume that $Q=k P$ is computed so that $k$ is a secret nonce and $P$ may or may not be known by the attacker. Let $E\left(\mathbb{F}_{q}\right)$ be an additive abelian group of points on an elliptic curve, where $\mathbb{F}_{q}$ is the finite field of $q$ elements. The group $E\left(\mathbb{F}_{q}\right)$ is formed by the points that satisfy the curve equation either including or appended with the point-at-infinity $\infty$, which is the zero element of the group.

We consider scalar multiplication algorithms that compute $k P$ by using precomputations on $P$. We show the general structure of these algorithms in Alg. 1 . The secret scalar is converted by using a recoding transformation $\Delta(k)$ into $\ell$ digits $d=\left(d_{0}, d_{1}, \ldots, d_{\ell-1}\right)$, where $d_{i} \in \mathcal{D}$ with $|\mathcal{D}|=n$. Let $T$ be a table of $n$ precomputed points ${ }^{1}: T_{t} \in E\left(\mathbb{F}_{q}\right)$ for all $t \in \mathcal{D}$. The table $T$ is computed from the base point $P$ via a precomputation transformation $\Pi(P)$. The bulk of the scalar multiplication is performed in a for-loop that iterates a target transformation $\Psi\left(R, T, d_{i}\right)$, where a static $T$ is used depending on the value of $d_{i}$. The

\footnotetext{
${ }^{1}$ For simplicity and without loss of generality, we consider one-to-one mapping between digit values $d_{i}$ and points in $T$. In practice, the precomputed table may not include all values; e.g., the values for negative digits can be derived on-the-fly, etc.
} 


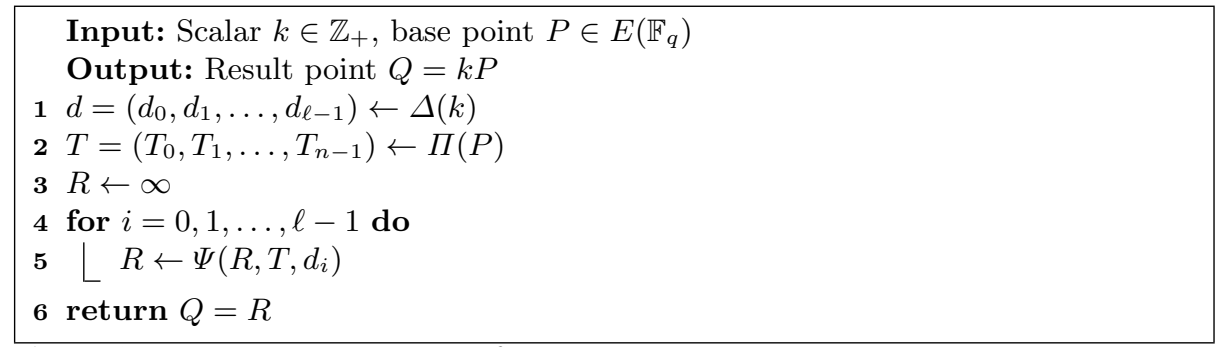

Algorithm 1: The structure of targeted scalar multiplication algorithms

attacker focuses only on transformation $\Psi$ and aims to find the value of $d_{i}$ by utilizing the fact that $\Psi$ uses the precomputed table $T$ according to the digit value $d_{i}$; i.e., most typically it adds $T_{d_{i}}$ to $R$. The attacker does not need access to the precomputation; e.g., the table $T$ can be stored in a ROM as in [47].

While Alg. 1 depicts an abstract structure, it captures many commonly used algorithms with precomputations. The most interesting algorithms for our attack are algorithms which utilize a regular pattern of operations and are, thus, immune to basic single-trace attacks such as SPA. In this paper, we consider Okeya et al.'s SPA-resistant width-w non-adjancent form (w-NAF) scalar multiplication [45] as an example of a vulnerable algorithm, but the attack applies to a large number of scalar multiplication algorithms (see below). For the SPAresistant $w$-NAF, the precomputation $\Pi(P)$ computes odd multiples of $P$ :

$$
T=\left(-\left(2^{w}-1\right) P, \ldots,-3 P,-P, P, 3 P, \ldots,\left(2^{w}-1\right) P\right) .
$$

In practice, only positive multiples can be precomputed and negative multiples computed as a part of $\Psi$, but this does not have an effect on the attacks. The recoding $\Delta(k)$ finds $d$ with $d_{i} \in \mathcal{D}=\left\{ \pm 1, \pm 3, \ldots, \pm\left(2^{w}-1\right)\right\}$ such that $k=$ $\Delta^{-1}(d)=\sum_{i=0}^{\ell-1} d_{\ell-i-1} 2^{i w}$; details about $\Delta(k)$ are available in [45]. The target transformation is $\Psi\left(R, T, d_{i}\right)=2^{w} R+T_{d_{i}}$.

If the attacker knows the precomputed points that were used in $\Psi$ in all iterations of the for-loop, then she learns all $d_{i}$ and the secret value of $k$ simply with $k=\Delta^{-1}(d)$. Sect. 3 presents how she can find out $d_{i}$ if she (a) knows $T$ and (b) even if $T$ is unknown. In both cases, the attacker must know the algorithm and its parameters (e.g., $w, \ell$ and $n$ ), $\Delta^{-1}$, and certain implementation details of $\Psi$ (see Sects. 3-5).

A large number of scalar multiplication algorithm can be captured by Alg. 1 and are at least potentially vulnerable to our attacks. These include many nonregular algorithms such as double-and-add, (sliding) window (NAF) methods, etc. However, we are more interested about regular algorithms which are protected from SPA but can be vulnerable to our attacks. These include, e.g., regular $w$-NAF and $m$-ary methods $[20,34,40,45,51]$, regular width- $w \tau$-adic NAF method for Koblitz curves [46], the regular signed-digit comb methods [21,30], and scalar multiplications on curves with fast endomorphisms that use multiscalar multiplications with precomputations (e.g., GLV [24] and GLS [23] meth- 
ods and, in particular, the recent regular algorithm [19]). Some of these methods have been recently utilized in lightweight ECC implementations to achieve protection against single-trace attacks: e.g., [30] was used in [47], [46] in [48], and [19] in [14]. All deterministic countermeasures inside $\Psi$ such as atomicity of point addition and point doubling, unified addition formulae, etc., do not work against the attack and are, thus, also in the list of vulnerable methods when used in a scalar multiplication algorithm that utilizes precomputations in the above sense. Montgomery's ladder [41], Joye's regular algorithms [33], and other algorithms, where for-loops iterate only on non-static values, are not vulnerable because there is no static $T$ that would be used in $\Psi$ which is required by our attacks. However, other similar types of single-trace attacks have recently identified similar weaknesses also in such algorithms (e.g., $[4,5,31]$ ).

Because $k$ is a nonce, we focus only on single-trace attacks. However, the attacker is often able to retrieve $t$ side-channel traces with different scalars (e.g., by observing $t$ ECDSA signature generations). This allows her to carry out a repeated single-trace attack where she trials successive single-trace attacks until one of them succeeds. The probabilities work for the attacker. Let $p_{\text {sta }}$ be the success probability of a single-trace attack. Then, the success probability of a repeated single-trace attack as a function of $t$ is given by:

$$
p_{\text {r-sta }}(t)=1-\left(1-p_{\text {sta }}\right)^{t} .
$$

E.g., if $p_{\text {sta }}=2 \%$, the repeated single-trace attack succeeds with over $50 \%$ probability only after 35 traces and with over $99 \%$ probability after 228 traces. These numbers are completely realistic in many practical scenarios.

\section{Description of the Attack}

We extend the previous advanced single-trace attacks and, in particular, Walter's Big Mac [52] and Clavier et al.'s horizontal correlation [11] attacks on exponentiations to scalar multiplications with precomputations. Contrary to collision correlation attacks $[4,5]$, which make a decision of which operation was computed by correlating multiple values of a side-channel trace to find out whether the operations used the same operands, our attack relies on distinguishing which particular static precomputed value was used in an operation.

Our attack targets the transformation $\Psi$ and, especially, field multiplications in $\Psi$ which use values from $T$ as operands. We assume that the word-length $W$ of the processor is small $\left(W \ll \log _{2} q\right.$, e.g,. $W=8$ or $\left.W=16\right)$ so that elements of $\mathbb{F}_{q}$ split into multiple words. The words of an element $a \in \mathbb{F}_{q}$ are $a_{0}, a_{1}, \ldots, a_{N-1}$ where $N=\left\lceil\log _{2} q / W\right\rceil$. In particular, we are interested in partial multiplications of a long integer multiplication (multiprecision multiplication) similarly to, e.g., $[4,5,11,52]$. Let $a, b \in \mathbb{F}_{q}$. Then, $a \times b$ requires $N^{2} W \times W$-bit partial multiplications $a_{i} \times b_{j}$ with all $0 \leq i, j<N$. Although we focus on $W \times W$-bit partial multiplications of an integer multiplication, the attack can be straight-

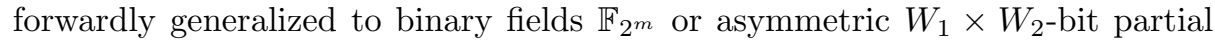


multiplications. It may also be possible to use other leakage sources including, e.g., memory addresses, register loads and stores, and multiprecision additions.

We assume that the attacker can identify all partial multiplications from a side-channel trace that belong to a long integer multiplication which uses a value from the precomputed table $T$ as one of the operands (say, $a$ ). The other operand $(b)$ is considered random ${ }^{2}$. The attacker collects samples from the partial multiplications. Let $s_{i, j}$ be a sample collected from the partial multiplication $a_{i} \times b_{j}$. The hypothesis is that $s_{i, j}$ depends on the properties of $a_{i}$ and $b_{j}$ (e.g., on their Hamming weights) and that utilizing this information allows the attacker to find out which value from $T$ was used as the operand $a$.

Following Walter's example [52], most of the effects of the unknown $b$ can be filtered out by computing an average over all samples that use $a_{i}$ :

$$
\hat{s}_{i}=\frac{1}{N} \sum_{j=0}^{N-1} s_{i, j} .
$$

Even if $b_{j}$ blinds $a_{i}$ in $s_{i, j}$ (e.g., because of a particularly low or high Hamming weight), computing the average efficiently reduces the dependence on the (unknown) value of $b$. The averaging acts also as a low-pass filter for filtering out measurement noise.

A single execution of $\Psi$ typically includes several multiplications with values from $T_{d_{i}}$ and information from all of them should be utilized in the attack. Let $M$ denote the number of such multiplications in $\Psi$. The attacker collects $\hat{s}_{0}^{i, m}, \hat{s}_{1}^{i, m}, \ldots, \hat{s}_{N-1}^{i, m}$ where $i$ is the index of $d_{i}$ and $m=0,1, \ldots, M-1$ is the index of the multiplication. She concatenates them into a sample vector that consists of $M N$ averaged samples ( $M N^{2}$ if not averaged):

$$
S_{i}=\left(\hat{s}_{0}^{i, 0}, \hat{s}_{1}^{i, 0}, \ldots, \hat{s}_{N-1}^{i, 0}, \hat{s}_{0}^{i, 1}, \ldots, \hat{s}_{N-1}^{i, 1}, \ldots, \hat{s}_{0}^{i, M-1}, \ldots, \hat{s}_{N-1}^{i, M-1}\right) .
$$

The attacker repeats the procedure for all $\ell$ executions of $\Psi$, after which she possesses $S_{0}, S_{1}, S_{2}, \ldots, S_{\ell-1}$ corresponding to digits $d_{0}, d_{1}, \ldots, d_{\ell-1}$, respectively. Next, we show two alternatives for finding out $d_{i}$ from the sample vectors.

\subsection{Correlation Attack with Known Precomputations}

We assume that the attacker knows $P$ and $\Pi$ which allows her to compute the precomputed table $T$. Ideally, the attacker would be able to profile a similar device and use the profile to produce accurate estimates for each value in $T$, i.e., to launch a template attack (see, e.g., $[2,16]$ for template attacks against ECC). In the following, we consider a model where the attacker only knows $T$ and is able to produce the sample vectors $S_{0}, S_{1}, S_{2}, \ldots, S_{\ell-1}$ from the measurements. The knowledge of $T$ is typically a weak assumption because $P$ is public in many protocols.

${ }^{2}$ If $R$ is not randomized in the beginning of Alg. $1, b$ is not random and even more powerful attacks are possible by considering also its value recursively during Alg. 1 . 
The attacker computes the table $T$. Based on its values, she calculates an estimate vector $E^{t}$ for each $t \in \mathcal{D}$. In this paper, we consider $E^{t}$ which are based on the Hamming weights of the words in $T$, but other methods can be used in a straightforward manner. As discussed above, $\Psi$ uses values from $T$ in $M$ multiplications. Let $T_{t}^{m}$ be the value that is used in multiplication $m$ if $d_{i}=t$. Let $H\left(T_{t, i}^{m}\right)$ denote the Hamming weight of the $i^{\text {th }}$ word of $T_{t}^{m}$. We set $e_{i}^{t, m}=H\left(T_{t, i}^{m}\right)$ and concatenate them with $i=0, \ldots, N-1$ and $m=0, \ldots, M-1$ into an estimate vector corresponding to digit value $d_{i}=t$ :

$$
E^{t}=\left(e_{0}^{t, 0}, e_{1}^{t, 0}, \ldots, e_{N-1}^{t, 0}, e_{0}^{t, 1}, \ldots, e_{N-1}^{t, 1}, \ldots, e_{0}^{t, M-1}, \ldots, e_{N-1}^{t, M-1}\right) .
$$

The attacker treats each sample vector $S_{i}$ individually by computing correlations between $S_{i}$ and $E^{t}$ with all $t \in \mathcal{D}$. Her digit guess $\delta_{i}$ for the value of $d_{i}$ is the value $t$ receiving the highest correlation:

$$
\delta_{i}=\underset{t \in \mathcal{D}}{\arg \max }\left(\operatorname{cor}\left(S_{i}, E^{t}\right)\right) .
$$

When all $i=0,1, \ldots, \ell-1$ have been analyzed similarly, the attacker has a scalar guess $\delta=\left(\delta_{0}, \delta_{1}, \ldots, \delta_{\ell-1}\right)$ and she can compute a trial scalar multiplication $Q^{\prime}=\kappa P$ with $\kappa=\Delta^{-1}(\delta)$. The attack is successful iff $Q^{\prime}=Q$.

\subsection{Clustering Attack with Unknown Precomputations}

We assume that the attacker is not able to construct $T$, e.g., because she does not know $P$, or $\Pi$ produces a randomized table $T$. Thus, she cannot calculate the estimates $E^{t}$ and is unable to launch the correlation attack of Sect. 3.1. We assume that the unknown table $T$ remains static for the entire scalar multiplication (e.g., randomization of $T$ happens only in $\Pi$ ). In that case, the same value from $T$ is used in $\Psi$ for two iterations $i$ and $j$ if $d_{i}=d_{j}$.

The attacker begins by acquiring sample vectors $S_{0}, S_{1}, S_{2}, \ldots, S_{\ell-1}$ according to (4). She then uses a clustering algorithm, which puts each of them into a cluster in $\mathcal{C}=\{1,2, \ldots, n\}$, and obtains a cluster vector $C=\left(c_{0}, c_{1}, \ldots, c_{\ell-1}\right)$ where $c_{i} \in \mathcal{C}$. If the clustering was made correctly, then $c_{i}=c_{j}$ iff $S_{i}$ and $S_{j}$ used the same value from $T$ and, thus, $d_{i}=d_{j}$. In this paper, we use unsupervised $k$ means clustering, but other clustering algorithms can be straightforwardly used and some may lead to better results in practice.

Each cluster in $\mathcal{C}$ represents a digit value in $\mathcal{D}$, but the mapping $\pi: \mathcal{C} \rightarrow \mathcal{D}$ is unknown to the attacker. There are in total $n$ ! possible mappings and if $n$ is small, the attacker can trial all of them. She iterates over all possible $\pi$, finds $\delta=\left(\pi\left(c_{0}\right), \pi\left(c_{1}\right), \ldots, \pi\left(c_{\ell-1}\right)\right)$, and checks if $\kappa=\Delta^{-1}(\delta)$ is equal to $k$ until one of them is successful. If the clustering is correct, this process always returns the correct $\pi$. The attacker does not need to know specifics about the implementation of long integer multiplications in order to launch the clustering attack, because she can directly cluster sample vectors without the averaging of (3).

Clustering is a viable attack strategy only when $n$, the number of possible digits (clusters), is small. First, $n$ ! quickly becomes very large which eventually 
makes finding the correct $\pi$ impossible. E.g., $n !=16 ! \approx 2^{44.25}$ is hard, but possible, to brute-force, but $32 ! \approx 2^{117.66}$ is already completely impractical. Second, $\ell$, the number of digits in $d$, gets smaller when $n$ and $w$ grow. Hence, $n$ becomes close to $\ell$ and each digit value appears only few times (or not at all) in $d$ and clustering cannot gain much information about $d$. While the clustering attack is in general weaker than the correlation attack, it does not make any assumptions about the leakage of a device under attack. Hence, if the leakage model used for constructing $E^{t}$ is inaccurate, then the clustering attack may outperform the correlation attack.

Clustering has been previously used in side-channel attacks on ECC by Heyszl et al. in [31]. They used it for launching an attack without any profile about the device (a hardware implementation) by clustering repeating patterns in a power trace; i.e., they would cluster the entire trace of $\Psi$. In our case, the attacker is expected to have more information about the device and its operation as described above, which allows her to use clustering on processed samples.

\section{Simulations}

We consider the following simulation setup to study the feasibility of the attacks. The simulations were performed with Matlab R2015b. We consider scalar multiplications on NIST P-256 curve with the base point from [44]. We assume that the SPA-resistant $w$-NAF method from [45] is used so that one precomputes odd multiples $\pm P, \pm 3 P, \ldots, \pm\left(2^{w}-1\right) P$. We experiment with window widths $w=2,3,4,5$ and word sizes $W=8,16$. Because we consider negative points as part of $T$, the number of points in $T$ is $n=2^{w}$ and the table consists of $T_{t}=t P=\left(x_{t}, y_{t}\right)$ with $t \in \mathcal{D}=\left\{ \pm 1, \pm 3, \ldots, \pm\left(2^{w}-1\right)\right\}$. Digits $d_{i}$ are drawn uniformly at random from $\mathcal{D}$. The number of digits is $\ell=\lceil 256 / w\rceil=128,86,64,52$ for $w=2,3,4,5$, respectively.

The transformation $\Psi$ consists of $w$ point doublings followed by a point addition. The point addition $R \leftarrow R+T_{d_{i}}$ is the interesting part of $\Psi$. We assume that it is computed using mixed affine-Jacobian coordinates using the algorithm from [28], which is given in App. A. This algorithm includes two multiplications, $x_{2} \times t_{1}$ and $y_{2} \times t_{2}$, where $x_{2}$ and $y_{2}$ are values from $T$; i.e., $M=2$.

In the simulations, we construct the sample vectors by deriving a sample value $s_{i, j}=s_{i, j}^{(s)}+s_{i, j}^{(n)}$ for each partial multiplication $a_{i} \times b_{j}$. The operand $a_{i}$ is a $W$-bit word of a coordinate selected from $T$ by using $d_{i}$ (x-coordinate for the first multiplication and $y$-coordinate for the second). The operand $b_{j}$ is selected uniformly at random from $\left[0,2^{W}-1\right]$. The signal part is given by $s_{i, j}^{(s)}=H\left(a_{i} \times b_{j}\right)$, normalized to zero mean and unit variance $\left(\sigma_{s}^{2}=1\right)$. The measurement noise $s_{i, j}^{(n)}$ is white Gaussian noise with variance $\sigma_{n}^{2}$. The noise level $\sigma_{n}$ is incremented in steps of 0.1 . The noisy samples $s_{i, j}$ are averaged to $\hat{s}_{i}$ by using (3). The averaged samples from the two multiplications are concatenated into a sample vector $S_{i}$ corresponding to digit $d_{i}$ by using (4). The length of a sample vector $S_{i}$ is $M N=2 \cdot\lceil 256 / W\rceil$ which gives either 64 or 32 samples for $W=8$ and $W=16$, respectively. 


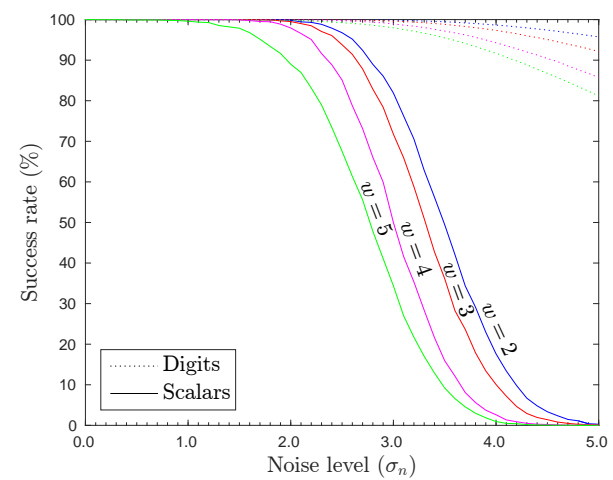

(a) $W=8$

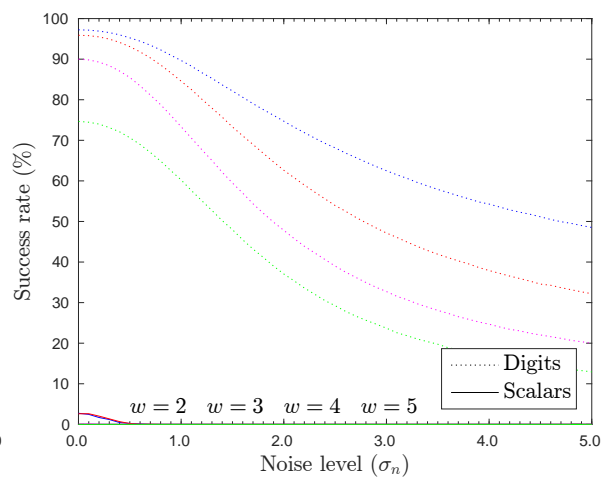

(b) $W=16$

Fig. 1. Results of the simulated correlation attack $(W=8$ and $W=16)$, averaged from 10,000 scalar multiplications for each noise level.

\subsection{Simulated Correlation Attack}

The correlation attack is simulated directly as described in Sect. 3.1.

The estimates $E^{t}$ are constructed by setting

$$
E^{t}=\left(H\left(x_{t, 0}\right), H\left(x_{t, 1}\right), \ldots, H\left(x_{t, N-1}\right), H\left(y_{t, 0}\right), H\left(y_{t, 1}\right), \ldots, H\left(y_{t, N-1}\right)\right),
$$

where $x_{t, i}$ and $y_{t, i}$ are $i^{\text {th }}$ word of the $x$ and $y$ coordinate of $T_{t}$, after which they are normalized to zero mean and unit variance. We use Pearson's linear correlation coefficient as the correlation function in (6).

Fig. 1 plots the results of the simulated correlation attack as a function of the noise level $\sigma_{n}$. It shows the success rates for individual digits $d_{i}$ and entire scalars $d$; i.e., which percentage of digits and $\ell$-digit scalars were guessed correctly. The digit success rates are only for evaluating the results of simulations, because in practice the attacker cannot verify the correctness of individual digits. The results are averages from 10,000 scalar multiplications (52,000-128,000 digits depending on the width $w$ ) for each noise level.

Fig. 1(a) shows that the correlation attack is extremely powerful with $W=8$ when noise is small: the attack works with $100 \%$ success rates for all $w$. Even high noise does not prevent an attack completely and allows a successful repeated single-trace attack as a consequence. The window width $w$ has a surprisingly small effect on the success rates. This is partly explained by the fact that $\ell$ gets smaller for larger $w$ which mitigates the effect of a lower digit success rate. On the other hand, Fig. 1(b) shows that the attack almost completely collapses when $W=16$. We see that attacks are successful only with low noise and, even then, with rather low success rates. However, even low success rates are enough to launch a successful repeated single-trace attack.

The leakage model $s_{i, j}^{(s)}=H\left(a_{i} \times b_{j}\right)$ used in Fig. 1 is quite pessimistic because the input operands do not have a direct contribution to the leakage, but only 


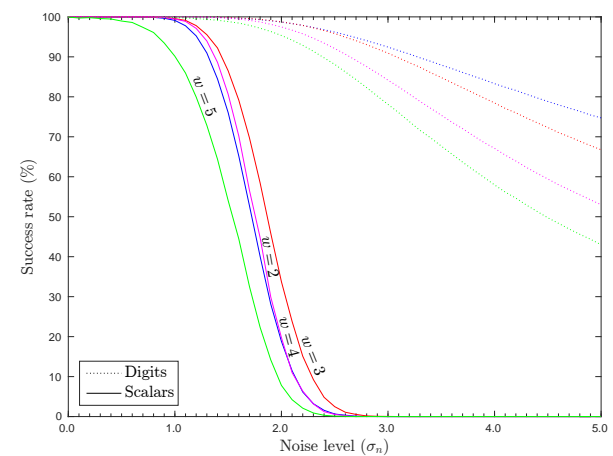

(a) $\omega=0.5$

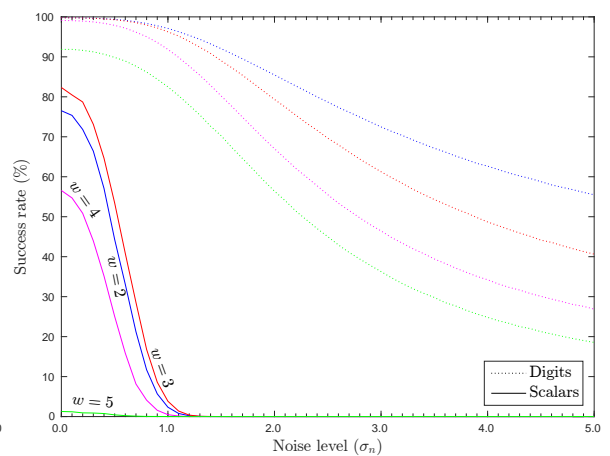

(b) $\omega=0.1$

Fig. 2. Results of the simulated correlation attack $(W=16)$ using the alternative leakage model with (a) $\omega=0.5$ and (b) $\omega=0.1$, averaged from 10,000 scalar multiplications for each noise level.

through the result of $a_{i} \times b_{j}$. Our experiments (see Sect. 5) hint that $a$ has a significantly stronger contribution to the leakage in practice. This motivated us to experiment with a different leakage model. We selected the following model $s_{i, j}^{(s)}=H\left(a_{i} \times b_{j}\right)+\omega\left(H\left(a_{i}\right)+H\left(b_{j}\right)\right)$; in this model, the term $H\left(a_{i} \times b_{j}\right)$ still dominates but $a$ has also a direct contribution through $H\left(a_{i}\right)$ with a weight $\omega$. Results with the alternative leakage model are shown in Fig. 2 for $\omega=0.5,0.1$. They show that if $H\left(a_{i}\right)$ contributes to the leakage directly even with a small weight, the correlation attack succeeds with high success rates for all $w$ even if $W=16$ (except $w=5$ when $\omega=0.1$ ).

\subsection{Simulated Clustering Attack}

Simulations of the clustering attack proceed similarly with the simulations of the correlation attack except that instead of computing correlations, we follow the blueprints of Sect. 3.2. In the simulation, we clustered $\ell$ sample vectors to $n$ clusters by using $k$-means clustering. We evaluated results of clustering in two ways. For $w \leq 3$, we performed a brute-force search through all $n$ ! possible $\pi$ (see Sect. 3.2). Even if this failed in finding the correct scalar (clustering was erroneous), we found the best $\pi$ by counting the number of correct digits. For $w=4$, brute-force is expensive and, hence, we only checked whether clustering was correct, i.e., whether all digits with the same values were clustered into same clusters (if this is the case, then the correct scalar can be found via brute-force). Because we lacked the best $\pi$, we did not provide digit success rates for $w=4$. In reality, the attacker needs to brute-force through $n$ ! possibilities in order to find whether the attack was a success. Fig. 3 collects results from simulations of 10,000 scalar multiplications per noise level $\sigma_{n}$ (in steps of 0.1 ) for $w=2,3,4$ and $W=8,16$. Width $w=5$ was not simulated because then $n=32$ and $n$ ! prevents the attack in practice (see Sect. 3.2). 


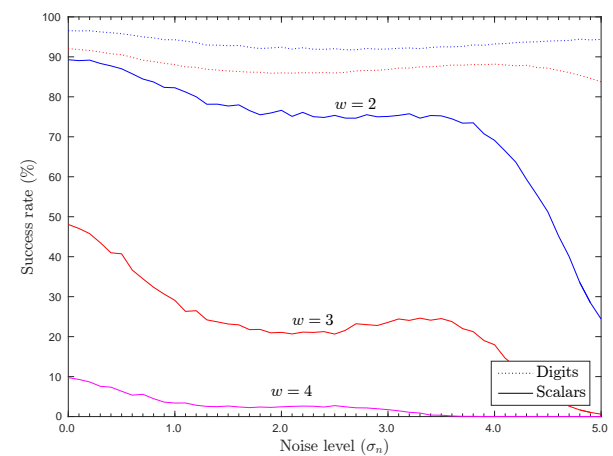

(a) $W=8$

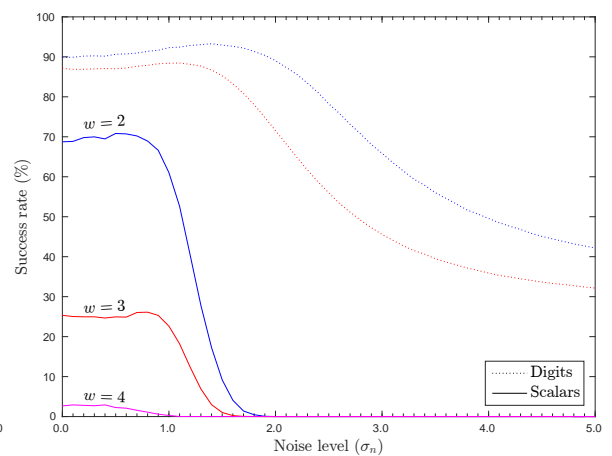

(b) $W=16$

Fig. 3. Results of the simulated clustering attack $(W=8$ and $W=16)$, averaged from 10,000 scalar multiplications for each noise level.

Fig. 3(a) presents the results for $W=8$ and shows that the clustering attack is successful for every $w$ but $w$ has a significantly larger effect than in the case of the correlation attack. Nevertheless, success rates are non-negligible for all $w$ (excluding $w=4$ and extreme noise) and (repeated) single-trace attacks are a threat. Noise has a significantly lower effect on the results compared to the correlation attack and the success rates remain flat for much of the noise spectrum. Despite being inferior to the correlation attack with low noise levels, the clustering attack outperforms correlation attack with high noise.

Fig. 3(b) shows that the clustering attack succeeds with relatively high success rates even when $W=16$ if noise is small. Most importantly, it outperforms the correlation attack with a large margin. As discussed in Sect. 4.1, the correlation attack fails to correlate leakage of the form $H\left(a_{i} \times b_{j}\right)$ with estimates $E^{t}$ based on the Hamming weights. On the other hand, clustering, which does not rely on a specific leakage model, is significantly more robust in this case. Although success rates of $W=16$ are surprisingly close to those of $W=8$ with low noise, higher noise levels prevent a successful attack significantly earlier.

\section{Experiments}

We selected for our experiments an 8-bit AVR microcontroller in a smart card body, a well-known platform commonly used in academic works to evaluate side channel attacks and countermeasures. Our particular model is an ATMega163 clocked at $4 \mathrm{MHz}$ and containing an $8 \times 8$-bit hardware multiplier that executes in two clock cycles. We implemented a semi-unrolled operand-scanning based multi-precision multiplication algorithm in assembly code, but the same results would be obtained, e.g., with product-scanning or operand-caching. Our code runs in constant-time and operates at byte level, i.e. for a word-length $W=8$. We monitored the power consumption via a $50 \Omega$ shunt resistor in the ground 
Table 1. Success rates from 200 emulated scalar multiplications using multiplication traces from the 8-bit AVR.

\begin{tabular}{|c|c|c|c|c|c|c|c|}
\hline $\begin{array}{l}\text { Window } \\
\text { width }\end{array}$ & $n$ & $\begin{array}{l}\text { Correlati } \\
\text { Digits }(\%)\end{array}$ & $\begin{array}{l}\text { attack } \\
\text { lars (\%) }\end{array}$ & $\begin{array}{c}\text { Clusterin } \\
\text { Digits (\%) }\end{array}$ & $\begin{array}{l}\operatorname{ack}^{\star} \\
\operatorname{rs}(\%)\end{array}$ & $\begin{array}{c}\text { Clustering } \\
\text { Digits (\%) }\end{array}$ & $\begin{array}{l}\operatorname{ck}^{\star, \diamond} \\
\operatorname{rs}(\%)\end{array}$ \\
\hline$w=2$ & 4 & 100.00 & 100.00 & 97.79 & 93.50 & 87.79 & 61.00 \\
\hline$w=3$ & 8 & 100.00 & 100.00 & 96.38 & 75.00 & 83.11 & 11.00 \\
\hline$w=4$ & 16 & 100.00 & 100.00 & $\mathrm{n} / \mathrm{a}$ & 41.50 & $\mathrm{n} / \mathrm{a}$ & 2.00 \\
\hline$w=5$ & 32 & 100.00 & 100.00 & $\mathrm{n} / \mathrm{a}$ & $\mathrm{n} / \mathrm{a}$ & $\mathrm{n} / \mathrm{a}$ & $\mathrm{n} / \mathrm{a}$ \\
\hline
\end{tabular}

* Success rates for $w=4$ are for correct clustering only

$\diamond$ No sample averaging in use (i.e., without (3))

path. The sampling rate of the oscilloscope was set at $250 \mathrm{MS} / \mathrm{s}$. We used the $\mathrm{I} / \mathrm{O}$ communication interface for triggering and synchronizing measurements.

We recorded traces from $256 \times 256$-bit multiplications, where one operand was random and the other was selected from the values of $T=( \pm P, \pm 3 P, \pm 5 P$, $\ldots, \pm 15 P)$, where $P$ is the NIST P-256 base point [44]. The power measurement traces were pre-processed to extract the points of interest, i.e. the intervals in which partial multiplications $a_{i} \times b_{j}$ were computed, by extracting the second cycle of each call to the MUL instruction. We further compressed data by taking the peak value of each clock cycle as a sample $s_{i, j}$ from a partial multiplication.

We emulated scalar multiplications with $w=2,3,4,5$ using the following procedure. We first selected $d$ by taking $\ell=\lceil 256 / w\rceil$ digits uniformly at random from $\mathcal{D}=\left\{1,3, \ldots,\left(2^{w}-1\right)\right\}$. Then, for all $d_{i}$, we constructed a trace representing an emulated point addition $R+T_{d_{i}}$ so that we randomly drew one trace which used $x_{d_{i}}$ as an operand from the set of measurements and another which used $y_{d_{i}}$ as an operand. These represented the interesting operations in $\Psi$ that our attack targets to (see App. A). Our emulated trace for one scalar multiplication thus consisted of $\ell$ such point addition traces built from two multiplication traces. We constructed traces for 200 emulated scalar multiplications using the above procedure by additionally ensuring that one multiplication trace was never used more than once in any of the emulated scalar multiplication traces.

The results of the experiments with emulated scalar multiplications are collected in Table 1. We provide the results of the clustering attack with and without sample averaging of (3). The latter reflects the situation where the attacker does not know the algorithm used for the long integer multiplication (and, thus, cannot use the averaging of (3)). The correlation attack works perfectly for all window widths: it is always capable of finding all 200 scalars $d$ correctly. The clustering attack is also able to find correct scalars with high success rates in every case when samples are averaged with (3). Even without this averaging the clustering attack is able to find at least a few correct scalars from 200 scalar multiplications for every $w$. Hence, a repeated single-trace attack would succeed in every case with few traces (see Sect. 2).

One distinctive observation from the results of Table 1 is that the clustering attack experiments outperform the simulated results shown in Fig. 3(a) even 
when simulations were performed with zero noise. A logical explanation for this is that the leakage model used in the simulations, where $s_{i, j}^{(s)}=H\left(a_{i} \times b_{j}\right)$, is too pessimistic. In practice $a$ appears to have a significantly larger effect on the leakage at least in the case of an 8-bit AVR (ATMega163).

\section{Countermeasures}

Given the results from the simulations and experiments, it is clear that both the correlation and clustering attack are potential threats also in practice. As discussed, e.g., in [5], Coron's three countermeasures against DPA attacks [13] do not offer protection against these single-trace attacks. Hence, more efficient countermeasures are needed in practice. Our attacks can be made significantly more difficult with the same countermeasures as other attacks of the same trend $[3,5]$. We survey existing countermeasures based on hiding and masking (particularly, from $[5,11])$ and discuss their effectiveness in the case of our attacks.

\subsection{Hiding}

The attacks require that the order of partial multiplications (a) is known by the attacker or (b) remains the same for the entire scalar multiplication for the correlation attack and the clustering attack, respectively. Randomizing this order serves as an effective countermeasure against both attacks (and other similar types of attacks). The order can be randomized in multiple ways (see [5] for different options) and the number of permutations can grow up to $N^{2}$ ! [5]. To avoid our attacks, it is essential to randomize the order of $a_{i}$ in the partial multiplications. This can be done up to $N$ ! permutations.

Randomizing the order of partial multiplications can lead to difficulties in efficient implementation of long integer multiplication because of carry propagation when accumulating different partial multiplications. E.g., randomizing the order prevents from using efficient long integer multiplication algorithms such as Comba's product-scanning algorithm [12]. If only the order of partial multiplications corresponding to a specific result word is randomized, then the low number of possible orders for low and high words may still allow successful attacks. Also, the amount of randomness may become a practical problem because the number of multiplications that needs to be randomized in one scalar multiplication is often large. Some of these technical issues were solved in [3].

\subsection{Masking}

Both attacks require that the table $T$ is static for one scalar multiplication (i.e., it does not change during the for-loop in Alg. 1). The correlation attack requires that $T$ is known by the attacker, which can be prevented by randomizing the entries of $T$ in $\Pi(P)$, e.g., by randomizing the base point by using Coron's countermeasure from [13]. However, the clustering attack will not be prevented 
with this because the table remains static for the for-loop and the computations of $\Psi$. The following masking thwarts both variants of the attack.

Let $r$ be a random value in $\mathbb{F}_{q}$. Then, we get the (additively) masked precomputed value by computing $a^{\prime}=a+r$. Multiplications are then computed by first computing $c^{\prime}=a^{\prime} \times b$ and second $c=c^{\prime}-r \times b$. Masking prevents the correlation attack because the attacker does not know the value of $a^{\prime}$. The precomputed values must be remasked for every multiplication (or at least several times in each scalar multiplication) to thwart the clustering attack. We must also ensure that $b$ is random; otherwise $r \times b$ may leak sensitive information. This can be achieved, e.g., by randomizing the base point.

Clavier et al. [11] considered a scheme where both operands are masked: $a^{\prime}=a+r_{1}$ and $b^{\prime}=b+r_{2}$. Then, $c^{\prime}=a^{\prime} \times b^{\prime}$ and $c=c^{\prime}-a \times r_{2}-b \times r_{1}-$ $r_{1} \times r_{2}$. This countermeasure does not apply in the case of our attacks: if fresh randomness is used for each multiplication, then it is possible to attack $a \times r_{2}$ and, if randomization of $a$ is done only in the beginning of a scalar multiplication (in $\Pi(P)$ ), then there is again no protection against the clustering attack.

Masking comes with a significant performance penalty because $M$ additional multiplications are required in each execution of $\Psi$. However, $M$ is often small (e.g., two). Another disadvantage is that the countermeasure requires large amounts of randomness $\left(\ell \cdot M \cdot\left\lceil\log _{2} q\right\rceil\right.$ bits per scalar multiplication).

\section{Conclusions and Future Work}

We studied single-trace side-channel attacks against scalar multiplications with precomputations. We presented two alternative approaches: one based on correlations and the other on clustering. We showed that they both pose a practical threat to scalar multiplication algorithms previously considered secure against single-trace attacks. In particular, implementations that use small word sizes are at risk. We demonstrated that it is possible to retrieve secret scalars from multiplications performed with an 8-bit AVR microcontroller (ATMega163). Our simulation results also show that at least implementations on 16-bit processors are potentially at risk. The future work includes studying the practical feasibility of the attacks with experiments on 16-bit and 32-bit processors $(W=16,32)$.

The simulations show that the correlation attack is sensitive to the type of leakage from the device. Consequently, leakage from integer multiplication should be studied more thoroughly in order to validate the feasibility of the (correlation) attack in processors with larger word sizes.

We considered the correlation and clustering attacks separately but they can be used also as a combination. E.g., the result from a correlation attack can be used as an initial clustering for a clustering attack or used in searching the correct mapping $\pi$ between clusters and digit values faster than brute-forcing through all possibilities.

If the attacker can select the base point $P$, then she can make sure that different entries in the table $T$ have distinctive fingerprints (e.g., very low Hamming weights for different words). Such an attack could be seen as an extension 
of refined power analysis [26] or doubling attack [22] to scalar multiplications with precomputations.

Future work includes research on methods that can find long-term secrets by utilizing partial information about the nonces, similarly to, e.g., [6]. For instance, how could the results of clustering help the attacker, when she knows which digits had same values with high probability (but with some errors in them). The case with correlations seems more straightforward because she can simply fix digits with the highest correlations and use methods similar to [6].

\section{Acknowledgments}

Parts of the work of Kimmo Järvinen were done when he was an FWO Pegasus Marie Curie Fellow in KU Leuven ESAT/COSIC and parts were supported by the INSURE project (303578) of Academy of Finland. This work was supported in part by the Research Council KU Leuven (C16/15/058), by the Flemish Government through FWO G.0550.12N, and by the Hercules Foundation AKUL/11/19. We thank Pekka Marttinen from Aalto University for discussions about clustering methods.

\section{A Point Addition Algorithm}

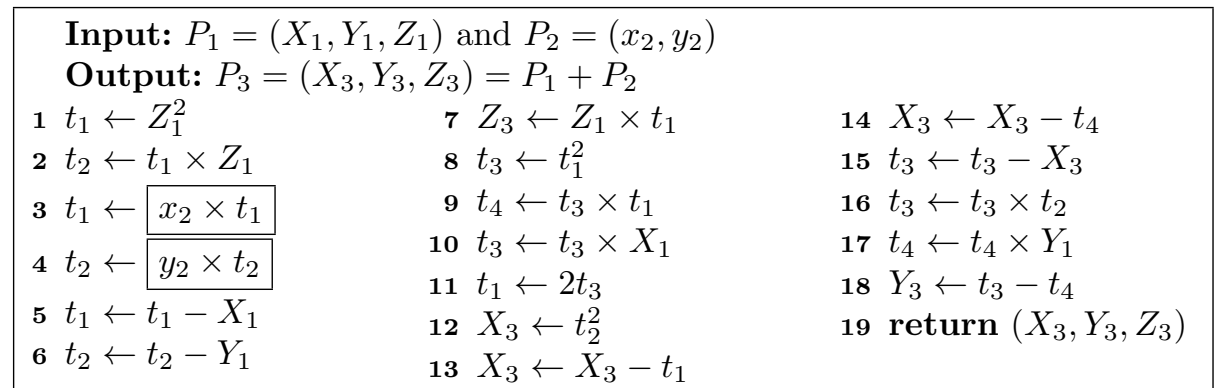

Algorithm 2: Point addition over $E\left(\mathbb{F}_{p}\right): y^{2}=x^{3}-3 x+b$ in affineJacobian coordinates [28]. The targeted multiplications are boxed.

\section{References}

1. Amiel, F., Feix, B., Villegas, K.: Power analysis for secret recovering and reverse engineering of public key algorithms. In: Selected Areas in Cryptography - SAC 2007. LNCS, vol. 4876, pp. 110-125. Springer (2007)

2. Batina, L., Chmielewski, Ł., Papachristodoulou, L., Schwabe, P., Tunstall, M.: Online template attacks. In: Progress in Cryptology - INDOCRYPT 2014. LNCS, vol. 8885, pp. 21-36. Springer (2014)

3. Bauer, A., Jaulmes, E., Prouff, E., Wild, J.: Horizontal and vertical side-channel attacks against secure RSA implementations. In: Topics in Cryptology - CT-RSA 2013. LNCS, vol. 7779, pp. 1-17. Springer (2013) 
4. Bauer, A., Jaulmes, E., Prouff, E., Wild, J.: Horizontal collision correlation attack on elliptic curves. In: Selected Areas in Cryptography - SAC 2013. LNCS, vol. 8282, pp. 553-570. Springer (2014)

5. Bauer, A., Jaulmes, E., Prouff, E., Reinhard, J.R., Wild, J.: Horizontal collision correlation attack on elliptic curves. Cryptography and Communications 7(1), 91119 (2015)

6. Benger, N., van de Pol, J., Smart, N.P., Yarom, Y.: "ooh aah... just a little bit": A small amount of side channel can go a long way. In: Cryptographic Hardware and Embedded Systems - CHES 2014. LNCS, vol. 8731, pp. 75-92. Springer (2014)

7. Bernstein, D., Birkner, P., Joye, M., Lange, T., Peters, C.: Twisted Edwards curves. In: Progress in Cryptology - AFRICACRYPT 2008. LNCS, vol. 5023, pp. 389405. Springer (2008)

8. Brier, E., Clavier, C., Olivier, F.: Correlation power analysis with a leakage model. In: Cryptographic Hardware and Embedded Systems - CHES 2004. LNCS, vol. 3156, pp. 16-29. Springer (2004)

9. Chevallier-Mames, B., Ciet, M., Joye, M.: Low-cost solutions for preventing simple side-channel analysis: Side-channel atomicity. IEEE Transactions on Computers 53(6), 760-768 (2004)

10. Chu, D., Großschädl, J., Liu, Z., Müller, V., Zhang, Y.: Twisted Edwards-form elliptic curve cryptography for 8-bit AVR-based sensor nodes. In: Proceedings of the 1st ACM Workshop on Asia Public-key Cryptography — AsiaPKC 2013. pp. 39-44. ACM (2013)

11. Clavier, C., Feix, B., Gagnerot, G., Roussellet, M., Verneuil, V.: Horizontal correlation analysis on exponentiation. In: Information and Communications Security — ICICS 2010. LNCS, vol. 6476, pp. 46-61. Springer (2010)

12. Comba, P.G.: Exponentiation cryptosystems on the IBM PC. IBM Systems Journal 29(4), 526-538 (1990)

13. Coron, J.S.: Resistance against differential power analysis for elliptic curve cryptosystems. In: Cryptographic Hardware and Embedded Systems - CHES 1999. LNCS, vol. 1717, pp. 292-302. Springer (1999)

14. Costello, C., Longa, P.: Four $\mathbb{Q}$ : Four-dimensional decompositions on a $\mathbb{Q}$-curve over the Mersenne prime. In: Advances in Cryptology — ASIACRYPT 2015, Part I. LNCS, vol. 9452, pp. 214-235. Springer (2015)

15. Danger, J.L., Guilley, S., Hoogvorst, P., Murdica, C., Naccache, D.: Improving the Big Mac attack on elliptic curve cryptography. Cryptology ePrint Archive, Report 2015/819 (2015)

16. Dugardin, M., Papachristodoulou, L., Najm, Z., Batina, L., Danger, J.L., Guilley, S., Courrege, J.C., Therond, C.: Dismantling real-world ECC with horizontal and vertical template attacks. In: Constructive Side-Channel Analysis and Secure Design - COSADE 2016. LNCS, vol. 9689, pp. 88-108. Springer (2016)

17. Fan, J., Verbauwhede, I.: An updated survey on secure ECC implementations: Attacks, countermeasures and cost. In: Naccache, D. (ed.) Cryptography and Security: From Theory to Applications: Essays Dedicated to Jean-Jacques Quisquater on the Occasion of His 65th Birthday. LNCS, vol. 6805, pp. 265-282. Springer (2012)

18. Fan, J., Gao, X., De Mulder, E., Schaumont, P., Preneel, B., Verbauwhede, I.: State-of-the-art of secure ECC implementations: a survey on known side-channel attacks and countermeasures. In: Proceedings of the 2010 IEEE International Symposium on Hardware-Oriented Security and Trust - HOST 2010. pp. 76-87 (2010) 
19. Faz-Hernández, A., Longa, P., Sánchez, A.H.: Efficient and secure algorithms for GLV-based scalar multiplication and their implementation on GLV-GLS curves (extended version). Journal of Cryptographic Engineering 5(1), 31-52 (2015)

20. Feix, B., Verneuil, V.: There's something about m-ary. In: Progress in Cryptology - INDOCRYPT 2013. LNCS, vol. 8250, pp. 197-214. Springer (2013)

21. Feng, M., Zhu, B.B., Zhao, C., Li, S.: Signed MSB-set comb method for elliptic curve point multiplication. In: Proceedings of the 2nd International Conference Information Security Practice and Experience - ISPEC 2006. LNCS, vol. 3903, pp. 13-24. Springer (2006)

22. Fouque, P.A., Valette, F.: The doubling attack-why upwards is better than downwards. In: Cryptographic Hardware and Embedded Systems - CHES 2003. pp. 269-280. LNCS, Springer (2003)

23. Galbraith, S.D., Lin, X., Scott, M.: Endomorphisms for faster elliptic curve cryptography on a large class of curves. Journal of Cryptology 24(3), 446-469 (2010)

24. Gallant, R.P., Lambert, R.J., Vanstone, S.A.: Faster point multiplication on elliptic curves with efficient endomorphisms. In: Advances in Cryptology - CRYPTO 2001. LNCS, vol. 2139, pp. 190-200. Springer (2001)

25. Giraud, C., Verneuil, V.: Atomicity improvement for elliptic curve scalar multiplication. In: Smart Card Research and Advanced Application - CARDIS 2010. LNCS, vol. 6035, pp. 80-101. Springer (2010)

26. Goubin, L.: A refined power-analysis attack on elliptic curve cryptosystems. In: Public Key Cryptography - PKC 2003. LNCS, vol. 2567, pp. 199-211. Springer (2002)

27. Gura, N., Patel, A., Wander, A., Eberle, H., Chang Shantz, S.: Comparing elliptic curve cryptography and RSA on 8-bit CPUs. In: Cryptographic Hardware and Embedded Systems - CHES 2004. LNCS, vol. 3156, pp. 119-132. Springer (2004)

28. Hankerson, D., Menezes, A.J., Vanstone, S.: Guide to elliptic curve cryptography. Springer Science \& Business Media (2004)

29. Hanley, N., Kim, H., Tunstall, M.: Exploiting collisions in addition chain-based exponentiation algorithms using a single trace. In: Topics in Cryptology - CTRSA 2015. LNCS, vol. 9048, pp. 431-448. Springer (2015)

30. Hedabou, M., Pinel, P., Bénéteau, L.: Countermeasures for preventing comb method against SCA attacks. In: Information Security Practice and Experience - ISPEC 2005, LNCS, vol. 3439, pp. 85-96. Springer (2005)

31. Heyszl, J., Ibing, A., Mangard, S., De Santis, F., Sigl, G.: Clustering algorithms for non-profiled single-execution attacks on exponentiations. In: Smart Card Research and Advanced Applications - CARDIS 2013. LNCS, vol. 8419, pp. 79-93. Springer (2014)

32. Heyszl, J., Mangard, S., Heinz, B., Stumpf, F., Sigl, G.: Localized electromagnetic analysis of cryptographic implementations. In: Topics in Cryptology - CT-RSA 2012. LNCS, vol. 7178, pp. 231-244. Springer (2012)

33. Joye, M.: Highly regular right-to-left algorithms for scalar multiplication. In: Cryptographic Hardware and Embedded Systems - CHES 2007. LNCS, vol. 4727, pp. 135-147. Springer (2007)

34. Joye, M.: Highly regular m-ary powering ladders. In: Selected Areas in Cryptography — SAC 2009. LNCS, vol. 5867, pp. 350-363. Springer (2009)

35. Joye, M., Yen, S.M.: The Montgomery powering ladder. In: Cryptographic Hardware and Embedded Systems - CHES 2002. LNCS, vol. 2523, pp. 291-302. Springer (2003)

36. Koblitz, N.: Elliptic curve cryptosystems. Mathematics of Computation 48(177), 203-209 (1987) 
37. Liu, Z., Seo, H., Großschädl, J., Kim, H.: Efficient implementation of NISTcompliant elliptic curve cryptography for 8-bit AVR-based sensor nodes. IEEE Transactions on Information Forensics and Security 11(7), 1385-1397 (2016)

38. Liu, Z., Wenger, E., Großschädl, J.: MoTE-ECC: Energy-scalable elliptic curve cryptography for wireless sensor networks. In: Applied Cryptography and Network Security - ACNS 2014. LNCS, vol. 8479, pp. 361-379. Springer (2014)

39. Miller, V.S.: Use of elliptic curves in cryptography. In: Advances in Cryptology CRYPTO '85. LNCS, vol. 218, pp. 417-426. Springer (1986)

40. Möller, B.: Securing elliptic curve point multiplication against side-channel attacks. In: Proceedings of the 4th International Conference Information Security - ISC 2001. LNCS, vol. 2200, pp. 324-334. Springer (2001)

41. Montgomery, P.L.: Speeding the Pollard and elliptic curve methods of factorization. Mathematics of Computation 48(177), 243-264 (1987)

42. Moradi, A., Mischke, O., Eisenbarth, T.: Correlation-enhanced power analysis collision attack. In: Cryptographic Hardware and Embedded Systems - CHES 2010. LNCS, vol. 6225, pp. 125-139. Springer (2010)

43. Nascimento, E., López, J., Dahab, R.: Efficient and secure elliptic curve cryptography for 8-bit AVR microcontrollers. In: Proceedings of the 5th International Conference on Security, Privacy, and Applied Cryptography Engineering - SPACE 2015. LNCS, vol. 9354, pp. 289-309. Springer (2015)

44. National Institute of Standards and Technology (NIST): Digital signature standard (DSS). Federal Information Processing Standard, FIPS PUB 186-4 (Jul 2013)

45. Okeya, K., Takagi, T.: The width-w NAF method provides small memory and fast elliptic scalar multiplications secure against side channel attacks. In: Topics in Cryptology - CT-RSA 2003. LNCS, vol. 2612, pp. 328-343. Springer (2003)

46. Okeya, K., Takagi, T., Vuillaume, C.: Efficient representations on Koblitz curves with resistance to side channel attacks. In: Information Security and Privacy ACISP 2005. LNCS, vol. 3574, pp. 218-229. Springer (2005)

47. Pessl, P., Hutter, M.: Curved tags - a low-resource ECDSA implementation tailored for RFID. In: Radio Frequency Identification: Security and Privacy Issues RFIDSec 2014. LNCS, vol. 8651, pp. 156-172. Springer (2014)

48. Sinha Roy, S., Järvinen, K., Verbauwhede, I.: Lightweight coprocessor for Koblitz curves: 283-bit ECC including scalar conversion with only 4300 gates. In: Cryptographic Hardware and Embedded Systems - CHES 2015. LNCS, vol. 9293, pp. 102-122. Springer (2015)

49. Specht, R., Heyszl, J., Kleinsteuber, M., Sigl, G.: Improving non-profiled attacks on exponentiations based on clustering and extracting leakage from multi-channel high-resolution EM measurements. In: Constructive Side-Channel Analysis and Secure Design - COSADE 2015. LNCS, vol. 9064, pp. 3-19. Springer (2015)

50. Szczechowiak, P., Oliveira, L.B., Scott, M., Collier, M., Dahab, R.: NanoECC: Testing the limits of elliptic curve cryptography in sensor networks. In: Proceedings of the 5th European Conference on Wireless Sensor Networks - EWSN 2008. LNCS, vol. 4913, pp. 305-320. Springer (2008)

51. Thériault, N.: SPA resistant left-to-right integer recodings. In: Selected Areas in Cryptography — SAC 2005. LNCS, vol. 3897, pp. 345-358. Springer (2006)

52. Walter, C.D.: Sliding windows succumbs to Big Mac attack. In: Cryptographic Hardware and Embedded Systems — CHES 2001. LNCS, vol. 2162, pp. 286-299. Springer (2001) 